\title{
Deformable model based 3-D Neuron Tracing and Reconstruction from Microscope Images and Software System with Parallel GPU Implementation
}

\author{
Y. Wang, * B. Roysam ** \\ * ECSE Department, Rensselaer Polytechnic Institute, Troy, NY 12180 \\ ** ECE Department, University of Houston, Houston, Texas 77204
}

The problem of accurately tracing axonal and dendritic arbors imaged with light microscopy is vital to comprehensive characterization of neuronal morphology ${ }^{2}$. Manual digital reconstructions from images produced by brightfield or fluorescence microscopy are far too labor intensive since dendritic and axonal arbors are large and complex. As illustrated in Figure 1A, the image stack which was acquired with 2-photon lasers scanning microscopy at the Neocortical layer 1 region is noisy and contains entangled axons. Figure 2A shows another image stack acquired with confocal microscopy at the peripheral neuromuscular region. The axons in this image are twisted and close-lying. Despite considerable progress in the field and the advent of several academic and commercial systems, the state of the art has remained unsatisfactory. Most algorithms in the literature focus on limited collections of image available internally to select groups and are not applicable to novel images. This motivated the DIADEM Challenge ${ }^{3}$ to stimulate further progress. The proposed methods were developed in response to this challenge. We developed a broadly applicable and fully automatic framework $^{1}$ and an open-source software system for tracing and reconstructing neurons from fluorescence microscope images. It is able to extract the morphological representation consisting of the centerlines of axons and the branching points (Figure 1BE) fully automatically. It provides finer segmentation result in which axons are segmented (Figure 1CF) and have unique labels (Figure 2(B-F)), and provides surface reconstruction result represented in geometric meshes (Figure 1G).

Our open-source tracing software system is a combination of visualizations, automated tracing, interactive inspection and editing functionalities. In the system, a set of seed points are detected from the original 3-D image. They are used to initialize a set of deformable models (3-D open-curve snake) which fit into the axons using image evidence and prior knowledge. The tracing result provides a tree representation consisting of centerlines and branching points (Figure 1BE). To extract more measurement and for better visualization, the system provides an approach based on Fast Marching to perform finer segmentation of neurons after tracing. The finer segmentation result is either a 3-D 2-label image in which neurites are separated from the background (Figure 1CF), or a 3-D multilabel image in which axons are separated and each has its unique label (Figure 2(B-F)). It can be seen from Figure 2(B-F) that our system produced accurate axon reconstruction result even for axons that are twisted and close-lying. In the software system, we implemented some computationally intensive steps (e.g. gradient vector flow (GVF)) on the NVIDIA Quadro FX4800 graphics processor, achieving an $18 \times$ speedup.

\section{References}

[1] Y. Wang et al., Neuroinformatics (in press) 2011.

[2] K.M. Brown et al., Neuroinformatics, Jan 2011.

[3] Diadem Challenge, http://diademchallenge.org/

[4] This work was supported by NIH Grant R01 EB005157. 

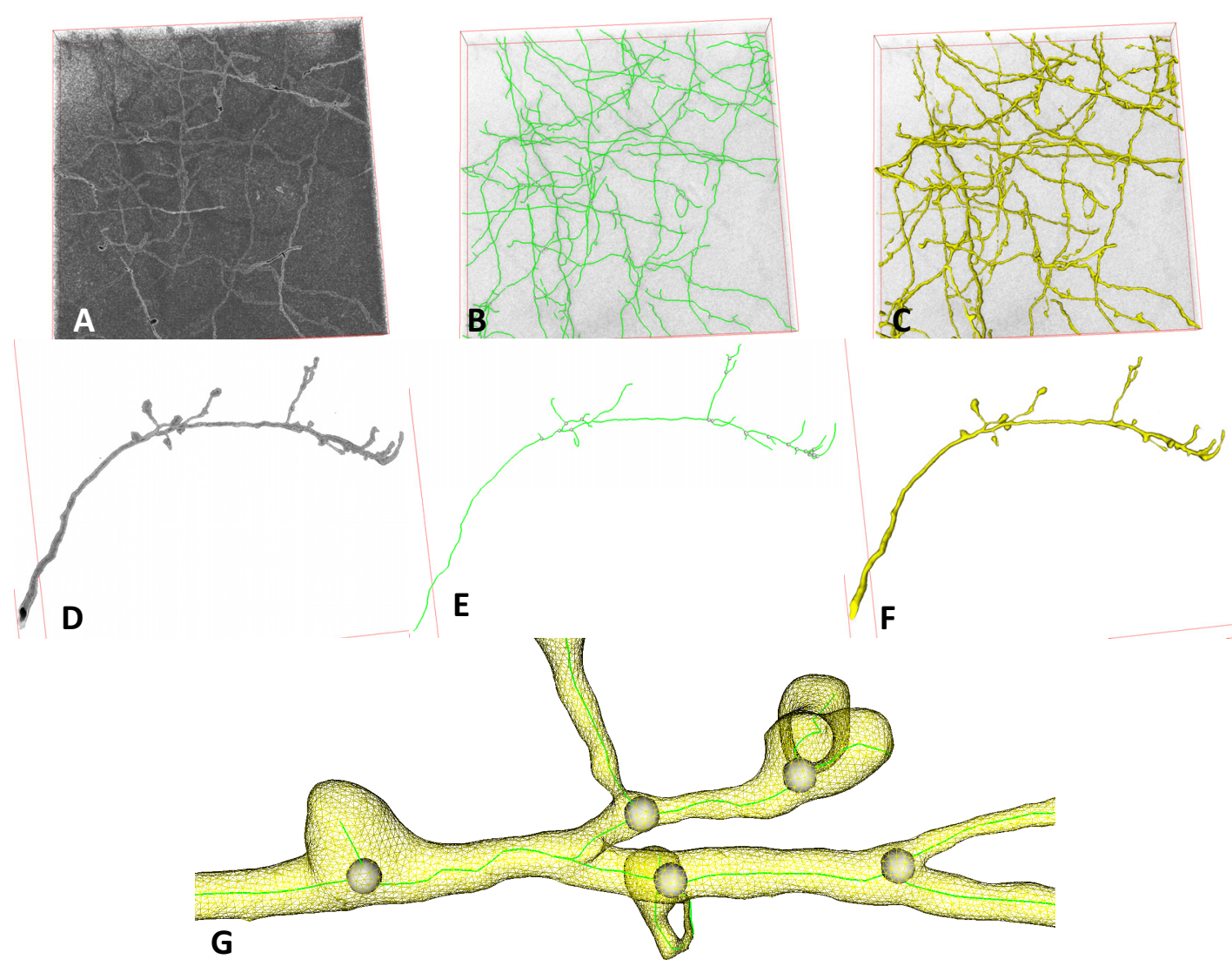

FIG. 1. (A-C) and (D-F) illustrate two examples of tracing image stacks from the Neocortical and Olfactory Projection Fiber dataset. (A)/(D) shows the original noisy image, (B)/(E) shows the tracing result (green lines) and $(\mathrm{C}) /(\mathrm{F})$ shows the segmentation result. $(\mathrm{G})$ shows the neuron reconstruction result. The surface is represented as 3-D mesh. The white spheres denote the branching points.

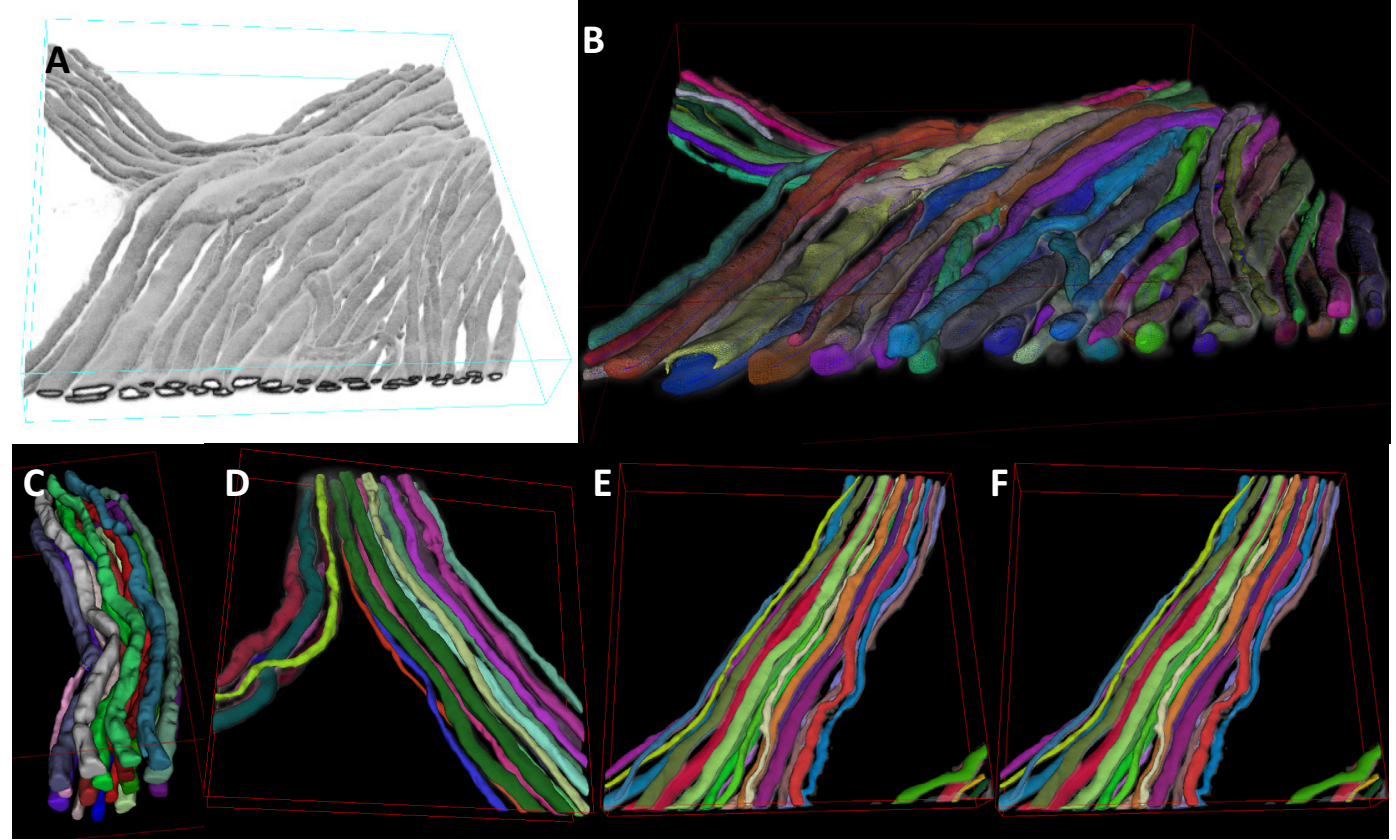

FIG. 2. (A) Illustrating one image stack from the Neuromuscular Projection Fiber dataset. The axons are twisted and close-lying. (B) Illustrating the axon reconstruction result. The identities of axons are color-coded. (C-F) More examples showing the axon reconstruction results by our method. 